\title{
Messier 35 (NGC 2168) DANCe
}

\section{Membership, proper motions, and multiwavelength photometry ${ }^{\star}$}

\author{
H. Bouy ${ }^{1}$, E. Bertin ${ }^{2}$, D. Barrado ${ }^{1}$, L. M. Sarro ${ }^{3}$, J. Olivares ${ }^{3,4,5}$, E. Moraux ${ }^{4,5}$, J. Bouvier ${ }^{4,5}$, J.-C. Cuillandre ${ }^{6}$, \\ Á. Ribas ${ }^{1,7,8}$, and Y. Beletsky ${ }^{9}$ \\ 1 Centro de Astrobiología, depto de Astrofísica, INTA-CSIC, PO Box 78, ESAC Campus, Villanueva de la Cañada, \\ 28691 Madrid, Spain \\ e-mail: hbouy@cab.inta-csic.es \\ 2 Institut d'Astrophysique de Paris, CNRS UMR 7095 and UPMC, 98bis Bd Arago, 75014 Paris, France \\ 3 Dpt. de Inteligencia Artificial, UNED, Juan del Rosal 16, 28040 Madrid, Spain \\ ${ }^{4}$ University Grenoble Alpes, IPAG, 38000 Grenoble, France \\ 5 CNRS, IPAG, 38000 Grenoble, France \\ ${ }^{6}$ Canada-France-Hawaii Telescope Corporation, 65-1238 Mamalahoa Highway, Kamuela, HI96743, USA \\ 7 European Space Astronomy Centre (ESA), PO Box 78, 28691 Villanueva de la Cañada, Madrid, Spain \\ 8 ISDEFE - ESAC, PO Box 78, 28691 Villanueva de la Cañada, Madrid, Spain \\ 9 Las Campanas Observatory, Carnegie Institution of Washington, Colina el Pino, 601 Casilla, La Serena, Chile
}

Received 12 December 2014 / Accepted 18 January 2015

\section{ABSTRACT}

\begin{abstract}
Context. Messier 35 (NGC 2168) is an important young nearby cluster. Its age, richness and relative proximity make it an ideal target for stellar evolution studies. The Kepler K2 mission recently observed it and provided a high accuracy photometric time series of a large number of sources in this area of the sky. Identifying the cluster's members is therefore of high importance to optimize the interpretation and analysis of the Kepler $\mathrm{K} 2$ data.

Aims. We aim to identify the cluster's members by deriving membership probabilities for the sources within $1^{\circ}$ of the cluster's center, which is farther away than equivalent previous studies.

Methods. We measure accurate proper motions and multiwavelength (optical and near-infrared) photometry using ground-based archival images of the cluster. We use these measurements to compute membership probabilities. The list of candidate members from the literature is used as a training set to identify the cluster's locus in a multidimensional space made of proper motions, luminosities, and colors.

Results. The final catalog includes 338892 sources with multiwavelength photometry. Approximately half (194 452) were detected at more than two epochs and we measured their proper motion and used it to derive membership probability. A total of 4349 candidate members with membership probabilities greater than $50 \%$ are found in this sample in the luminosity range between 10 mag and 22 mag. The slow proper motion of the cluster and the overlap of its sequence with the field and background sequences in almost all color-magnitude and color-color diagrams complicate the analysis and the contamination level is expected to be significant. Our study, nevertheless, provides a coherent and quantitative membership analysis of Messier 35 based on a large fraction of the best ground-based data sets obtained over the past 18 years. As such, it represents a valuable input for follow-up studies using, in particular, the Kepler $\mathrm{K} 2$ photometric time series.
\end{abstract}

Key words. proper motions - stars: kinematics and dynamics - open clusters and associations: individual: NGC 2168

\section{Introduction}

Different galactic environments, it is assumed, produce distinct populations during the star formation episodes. From very loose and young associations such as Taurus-Auriga, doomed to be dispersed very soon in the galactic gravitational well, to the very old halo population in massive globular clusters, there is a large gamut in ages, compactness, metallicity, internal dynamics, total mass, spatial distribution and distribution of the individual masses, perhaps indicating differences in the initial mass functions (IMF). It is clear that a significant improvement in the completeness of the census of different associations provides key information to understanding star formation and evolution.

* Table 3 is only available at the CDS via anonymous ftp to cdsarc.u-strasbg.fr (130.79.128.5) or via

http://cdsarc.u-strasbg.fr/viz-bin/qcat?J/A+A/575/A120
In this context, the open cluster Messier 35 (NGC 2168, hereafter M 35) is very interesting in several aspects. First, it is a quasi-twin of the Pleiades cluster, with a similar age (Vidal 1973) but a different metallicity. Second, the Kepler K2 mission (Howell et al. 2014) has just provided exquisite photometric time series, so that a detailed distribution of the rotational periods for different masses will soon become available (e.g. Meibom et al. 2009). Moreover, M 35 is not so crowded that confusion is a major problem and its size (about $1 \mathrm{sq}$. deg) is very suitable for follow-up multiobject spectroscopy (Barrado et al. 2001a).

Several studies have provided estimates of other key cluster parameters, such as its age, census, and metallicity. Table 1 gives an overview of the cluster's properties found in the literature in the past 15 years. However, there is no a clear consensus for most of them. Sung \& Bessell (1999) derived a distance modulus of $(m-M)_{0}=9.60 \pm 0.10$ corresponding to $832 \mathrm{pc}$, 
Table 1. Overview of previous studies of M35.

\begin{tabular}{|c|c|c|c|c|}
\hline Reference & $\begin{array}{l}\text { Distance } \\
(\mathrm{pc})\end{array}$ & $\begin{array}{l}\text { Age } \\
(\mathrm{Myr})\end{array}$ & $\begin{array}{c}E(B-V) \\
(\mathrm{mag})\end{array}$ & {$[\mathrm{Fe} / \mathrm{H}]$} \\
\hline Reimers \& Koester (1988) & & $70-100$ & & \\
\hline Sung \& Bessell (1999) & $832 \pm 39$ & $200_{-100}^{+200}$ & $0.255 \pm 0.024$ & \\
\hline Barrado (2001) & & 180 & & \\
\hline Barrado et al. (2001a) & & $>125$ & & $-0.21 \pm 0.10$ \\
\hline Kalirai et al. (2003) & $912_{-65}^{+70}$ & 180 & & \\
\hline Steinhauer \& Deliyannis (2004) & & & & $-0.143 \pm 0.014$ \\
\hline Meibom et al. (2009) & & $134-161$ & & \\
\hline Geller et al. (2010) & & 133 & & \\
\hline McNamara et al. (2011) & $732 \pm 145$ & & & \\
\hline
\end{tabular}

Table 2. Instruments used in this study.

\begin{tabular}{|c|c|c|c|c|c|c|c|c|}
\hline Observatory & Instrument & Filters & $\begin{array}{c}\text { Platescale } \\
{\left[\text { pixel }^{-1}\right]}\end{array}$ & Chip layout & Chip size & Field of view & $\operatorname{Epoch}(s)$ & Ref. \\
\hline CFHT & UH $8 \mathrm{~K}$ & $R, I$ & 0.205 & $4 \times 2$ & $2 \mathrm{k} \times 4 \mathrm{k}$ & $29^{\prime} \times 29^{\prime}$ & 1996 & 1 \\
\hline CFHT & CFHT $12 \mathrm{~K}$ & $B, V, R, I, z$ & 0.201 & $6 \times 2$ & $2 \mathrm{k} \times 4 \mathrm{k}$ & $42^{\prime} \times 28^{\prime}$ & 1999, 2000, 2001, 2002 & 2 \\
\hline CFHT & MegaCam & $g, r$ & 0.187 & $4 \times 9$ & $2 \mathrm{k} \times 4 \mathrm{k}$ & $1^{\circ} \times 1^{\circ}$ & $2003,2005,2011$ & 3 \\
\hline CFHT & WIRCam & $\mathrm{H} 2$ & $0 ! 304$ & $2 \times 2$ & $2 \mathrm{k} \times 2 \mathrm{k}$ & $20^{\prime} \times 20^{\prime}$ & 2008, 2009, 2010, 2011 & 4 \\
\hline Subaru & Suprime-Cam & VR-Broad & 0.200 & $5 \times 2$ & $2 \mathrm{k} \times 4 \mathrm{k}$ & $34^{\prime} \times 27^{\prime}$ & 2005 & 5 \\
\hline INT & WFC & $g, \mathrm{H} \alpha, \mathrm{He} \mathrm{I}, i, I, r, U, V$ & 0.333 & $3 \times 1+1$ & $2 \mathrm{k} \times 4 \mathrm{k}$ & $34^{\prime} \times 34^{\prime}$ & $2000,2002,2003,2005,2008,2009,2010$ & 6 \\
\hline KPNO (Mayall) & NEWFIRM & $J, H, K \mathrm{~s}$ & 0.400 & $2 \times 2$ & $2 \mathrm{k} \times 2 \mathrm{k}$ & $28^{\prime} \times 28^{\prime}$ & 2008 & 7 \\
\hline KPNO (Mayall) & MOSAIC1 & VR-broad & 0.26 & $4 \times 2$ & $2 \mathrm{k} \times 4 \mathrm{k}$ & $36^{\prime} \times 36^{\prime}$ & $2006,2009,2010,2011,2012$ & 8 \\
\hline KISO & $2 \mathrm{KCCD}$ & $B, V, R, I$ & $1 "$ 1"50 & $1 \times 1$ & $2 \mathrm{k} \times 2 \mathrm{k}$ & $50^{\prime} \times 50^{\prime}$ & 2002,2003 & 9 \\
\hline SDSS DR9 & SDSS camera & $u, g, r, i, z$ & 0.396 & $6 \times 5$ & $2 \mathrm{k} \times 2 \mathrm{k}$ & $3^{\circ}$ & 2006 & 10 \\
\hline
\end{tabular}

References. (1) Metzger et al. (1995); (2) Cuillandre et al. (2000); (3) Boulade et al. (2003); (4) Puget et al. (2004); (5) Miyazaki et al. (2002); (6) Ives (1998); (7) Autry et al. (2003); (8) Wolfe et al. (2000); (9) Itoh et al. (2001); (10) Ahn et al. (2012).

a reddening of $E(B-V)=0.255 \pm 0.024 \mathrm{mag}$ and an age of $200_{-100}^{+200}$ Myr. Kalirai et al. (2003) pushed the cluster a little bit further, to $912_{-65}^{+70} \mathrm{pc}$ and derived an isochrone fitting age of 180 Myr. More recently, McNamara et al. (2011) obtained a distance of $732 \pm 145 \mathrm{pc}$ from the internal proper motions. This dynamical distance and the radial velocity dispersion obtained by Geller et al. (2010) imply an age of $133 \mathrm{Myr}$, according to the same authors. Meibom et al. (2009) performed an age determination based on a completely different technique, gyrochronology, yielding a value between $134 \mathrm{Myr}$ and 161 Myr. Reimers \& Koester (1988), by using the white dwarf cooling age, derived an age in the range 70-100 Myr, whereas Barrado (2001), with the same technique, estimated 180 Myr. The rotation pattern suggests an age slightly older than the Pleiades (175 Myr compared to $125 \mathrm{Myr}$ ). The same conclusion is reached when the lithium abundances are compared. This is because the lithium dispersion, which is itself related to rotation for a given stellar mass, is no longer obvious for M35 (Barrado et al. 2001a) while it is very significant in the case of the Pleiades (see, for instance Soderblom et al. 1993).

Regarding chemical composition, Steinhauer \& Deliyannis (2004) quoted an unpublished work with a metallicity $[\mathrm{Fe} / \mathrm{H}]=-0.143 \pm 0.014$, very similar to the value obtained by our group $([\mathrm{Fe} / \mathrm{H}]=-0.21 \pm 0.10)$ using high spectral resolution data and spectral synthesis, (Barrado et al. 2001a).

The cluster is also relatively massive: Leonard \& Merritt (1989) derived a central total mass in the range 1600-3200 $M_{\odot}$ using dynamical models. Our own estimate, for the central of $27.5 \times 27.5$ square arcmin, is $\sim 1600 M_{\odot}$, which allows for an exquisite determination of a complete stellar mass function (Barrado et al. 2001b, so far the deepest for M35) which is totally compatible with the IMF of much younger and unevolved clusters, such as such as Collinder 69 (Barrado et al. 2005; Bayo et al. 2011), and suggests that M 35 includes several tens of potential substellar members.

The goal of this paper is to complete the census reaching well inside the substellar domain and including the cluster outskirts up to a radius of $1^{\circ}$, providing multi-epoch, multiband photometry from public archives and from our recent observations.

\section{Data sets}

We searched the Canada France Hawaii Telescope (CFHT), National Optical Astronomical Observatory (NOAO), Subaru, Kiso, Sloan Digital Sky Survey (SDSS DR9, Ahn et al. 2012) and Isaac Newton Group (ING) public archives for wide field images of the cluster. Table 2 gives an overview of the data set found in these archives and in our own private archives.

In each case, we downloaded the raw data and associated calibration frames. We processed the individual raw images using an updated version of Alambic (Vandame 2002), a software suite developed and optimized for processing large multi-CCD imagers, which we adapted for the instruments used here. Alambic includes standard processing procedures, such as overscan, bias, and dark subtraction for the individual readout ports of each CCD, flat-field correction, bad pixel masking, CCD-to-CCD gain harmonization in the case of multichip instruments, fringing correction (when needed for the reddest filters), de-stripping, background subtraction, and nonlinearity correction in the case of near-infrared detectors.

Aperture, point spread function (PSF), and model photometry were extracted from the individual images using 


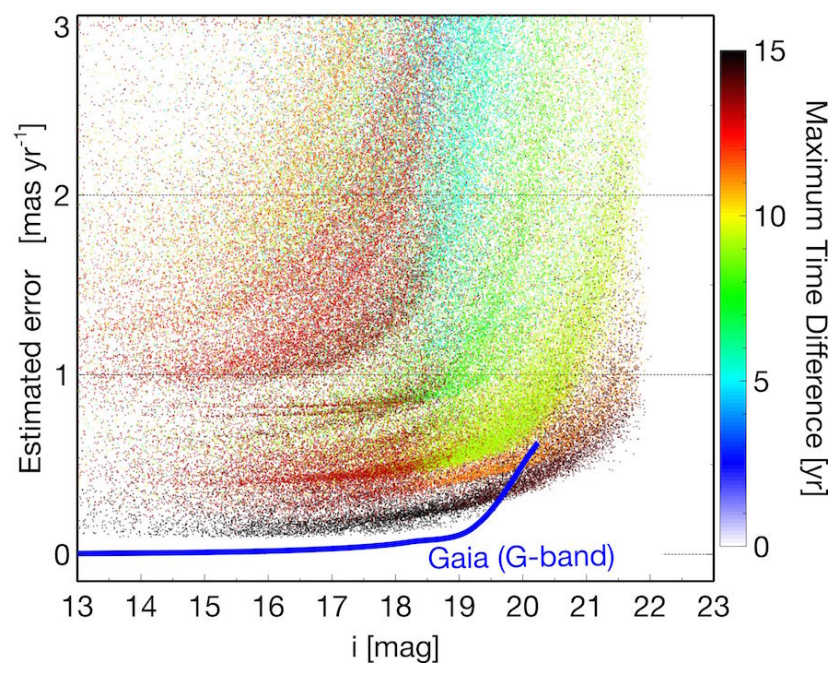

Fig. 1. Estimated error on the proper motion as a function of $i$-band magnitude. The color scale represents the maximum time difference used for each measurement. The prediction for Gaia is over-plotted for comparison.

SEXTRACTOR (Bertin \& Arnouts 1996) and PSFEX (Bertin 2011). The individual catalogs were then registered and aligned on the same photometric scale using ScAMP (Bertin 2006).

We derived the photometric zero-points using standard fields obtained for the same night whenever available or all-sky catalogues such as 2MASS (Skrutskie et al. 2006) in $J, H$ and $K$ s, SDSS in $g, r, i, z$ and IPHAS (Drew et al. 2005) in $\mathrm{H} \alpha$. The zeropoint rms were typically in the range between $0.02 \mathrm{mag}$ and $0.12 \mathrm{mag}$, and were added quadratically to the measurement uncertainty. We did not attempt to calibrate the nonstandard $V R$ filters used with the Mosaic and SuprimeCam cameras and the He I narrow-band filter obtained with the WFC.

Proper motions for all the sources were computed using the method described in Bouy et al. (2013). The final residuals on the astrometric solution show a dispersion on the order of $11 \sim 12$ mas. Figure 1 shows the estimated error on the proper motion as a function of $i$-band apparent magnitude. We note that the proper motion measurements are not tied to an absolute reference system such as the International Celestial Reference System (ICRS). They are nevertheless expected to be close to the ICRS since extragalactic sources and background stars largely dominate the sample and display very little motion with respect to the ICRS.

A final master catalog of all sources (338 892 in total) was then produced including the measured photometry in the grizJHKs filters. We detected a total of 194452 sources (approximately half) at more than two epochs, and the proper motions of these sources are also included. Since the main goal of the survey focuses on the study of M35, only objects with $\left|\mu_{\alpha} \cos \delta\right| \leq 100$ mas yr-1 and $\left|\mu_{\delta}\right| \leq 100$ mas yr$^{-1}$ were considered for the membership analysis.

\section{Membership}

We use the method described in Sarro et al. (2014) to derive membership probabilities of all the sources. Briefly, the method uses a training set - in this case the sample of candidate members from Barrado y Navascués et al. (2001) - to identify the features that most efficiently separate the cluster's population from the field and background population among all combinations of proper motion, luminosities and colors available. In the case

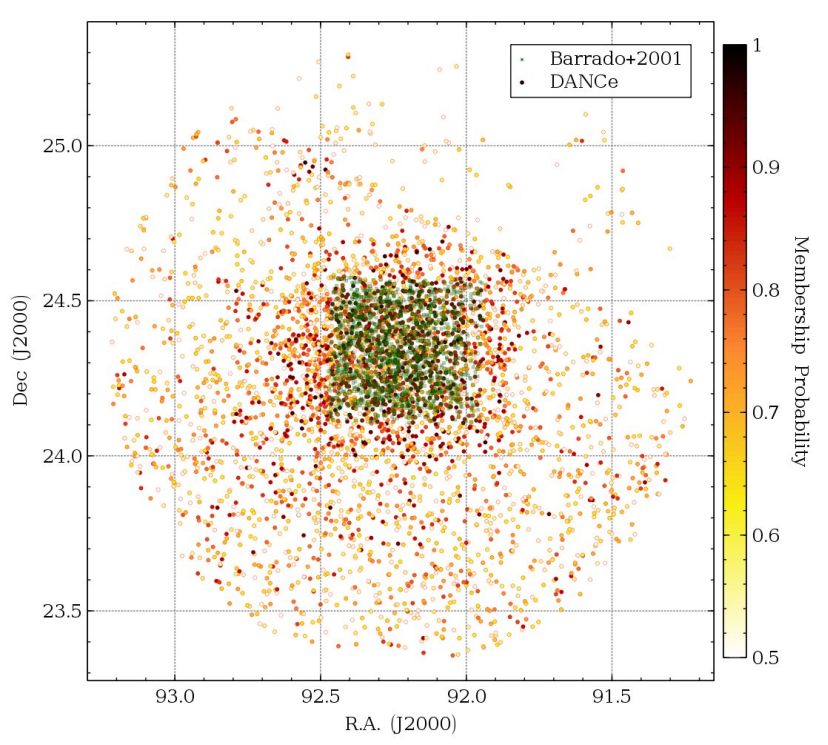

Fig. 2. Spatial distribution of sources used as training set (green squares, from Barrado y Navascués et al. 2001) and of the 4349 sources of the final sample with membership probability greater than 0.5. Background image: 2MASS $J$-band.

of M 35, the best set was found to include the proper motions, the $z, H, K$ s luminosities and the $(r-z),(r-i)$ and $(J-H)$ colors. Once the best set of colors, luminosities and proper motion is selected, a model of the cluster's locus defined by the training set is fitted in the corresponding multidimensional space. A mixture of Gaussians is used for the proper motion diagram and principal curves for the cluster's sequence in all color-magnitude and color-color diagrams. Membership probabilities are then computed using this first model. The highest probability members are selected as the new training set, and the models and the membership probabilities are recomputed until they converge.

This method was originally developed and optimized for clusters like the Pleiades, i.e. clusters with a relatively large proper motion and photometric sequence clearly separated from the field and background sequences in many color-magnitude diagrams. Unfortunately M 35 is a worst-case scenario for the method. Its proper motion is relatively small and its sequence largely overlaps with the field and background sequence in almost all color-magnitude and color-color diagrams. To limit the contamination and enhance the contrast between cluster members and field and background sources, we chose to restrict the analysis to a radius of $1^{\circ}$ around the cluster's center located at $\alpha=06^{\mathrm{h}} 08^{\mathrm{m}} 54.0^{\mathrm{s}}$, and $\delta=+24^{\circ} 20^{\prime} 00^{\prime \prime}(\mathrm{J} 2000)$. Figure 2 shows their spatial distribution. It shows that the northwestern part of the survey was shallower than the rest of the survey, as fewer members were selected. It also shows that a number of high probability members have $(r-i)$ or $(i-K \mathrm{~s})$ color apparently inconsistent with the cluster's sequence. Their high probability is due to their proximity to the isochrone in all other color-magnitude and color-color diagrams as well as to the cluster's locus in the proper motion diagram. These sources, probably suffering from inaccurate photometry in one of these bands, illustrate the robustness of the selection method based on a high dimensional space.

Table 3 gives the astrometry, photometry and membership probability for the 338892 unique sources detected in the corresponding area. Only the sources with a proper motion measurement have a membership probability (194452 in total), as the availability of a proper motion measurement was a necessary 

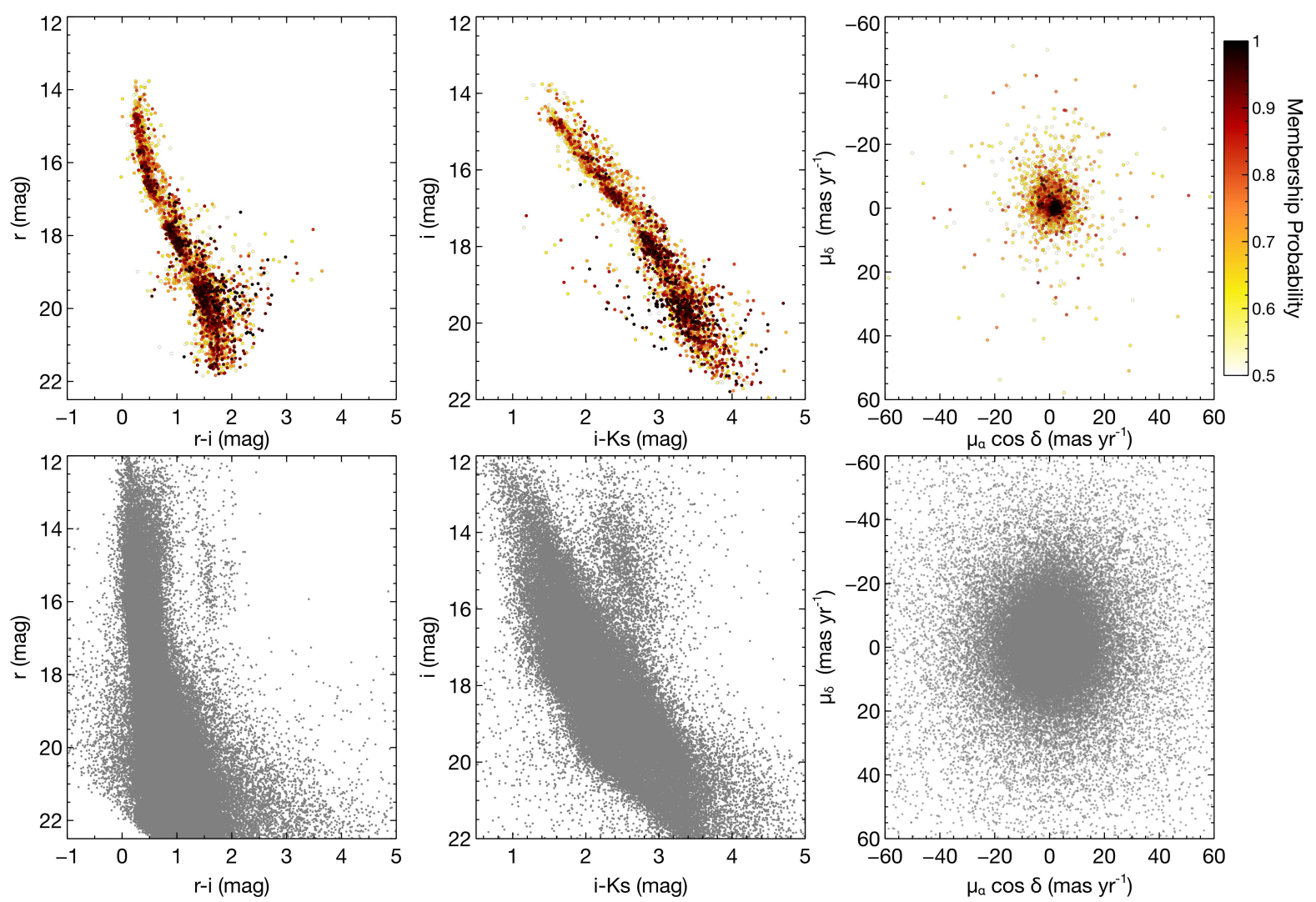

Fig. 3. Color magnitude and proper motion diagrams of the 194452 sources of the sample detected at more than two epochs. Left: $r$ vs. $r-i$. Middle: $i$ vs. $i-K$ s. Right: proper motion diagram. Sources with a membership probability greater or equal to 0.5 are represented in the top panels with a color scale proportional to their probability. The remaining sources are represented in the bottom panels.

condition in our membership analysis. Using sources with membership probability greater or equal to 0.9 , we derive a median proper motion for the cluster of $\left(\mu_{\alpha} \cos \delta, \mu_{\delta}\right)=(1.99,-0.23) \pm$ $(0.82,0.71)$ mas $^{-1} r^{-1}$, where the uncertainties correspond to the scaled median absolute deviation.

Figure 4 shows the membership distribution and cumulative distribution (computed from large to small values) for all the sources in the catalog. The vast majority of the sources has a membership probability of $\approx 0.0$. A monotonously decreasing distribution fills the probability range between 0 and 1.0 , as the result of the loose constraints on the photometric and astrometric properties of the cluster.

NGC 2158 is an intermediate age $(1 \sim 2$ Gyr $)$ cluster at a distance of $\approx 3600$ pc (Carraro et al. 2002) located at a projected distance of only $25^{\prime}$ from M 35 . It is therefore included in the area covered by the present study and possibly contaminates the membership analysis. To estimate the level of contamination due to NGC 2158 members, we select all the sources located within $3^{\prime}$ of its center. Given the distance and projected density profile of NGC 2158, the vast majority of these sources are expected to be members of NGC 2158. Figure 5 shows the sequence formed by these sources in various color-magnitude diagrams made of colors and luminosities used for the membership analysis presented here, and compares it to the sequence formed by objects with a probability of membership to M35 greater than 0.5 according to our analysis. The two sequences overlap mostly at the bright end, and contamination must occur mostly above $i \lesssim 17 \mathrm{mag}$. We find that only 27 sources with a probability of membership to M 35 greater than 0.5 are located within $3^{\prime}$ of NGC 2158, and 53 within $5^{\prime}$. Given the projected size of NGC 2158 core, little contamination is expected beyond $5^{\prime}$, and

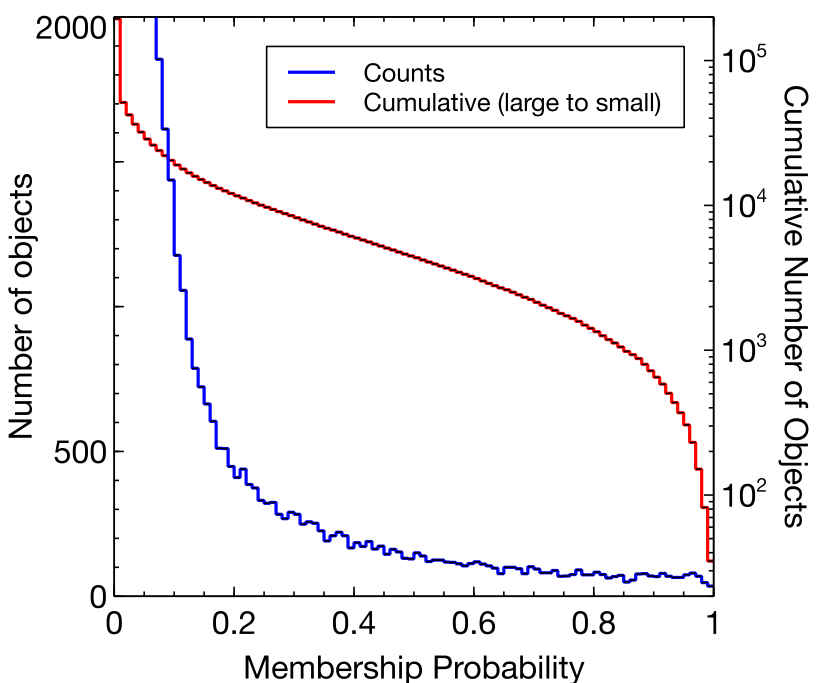

Fig. 4. Distribution (blue, left scale) and cumulative distribution (red, computed from large to small values, right scale) of membership probabilities for the 194452 sources of the sample.

the contamination by NGC 2158 members must not reach more than $1 \sim 2 \%$ at most.

\section{Conclusions}

We have processed and analyzed a total of 4867 wide-field optical and near-infrared images of M 35 obtained from various public astronomical archives. The images cover 18 years of time 

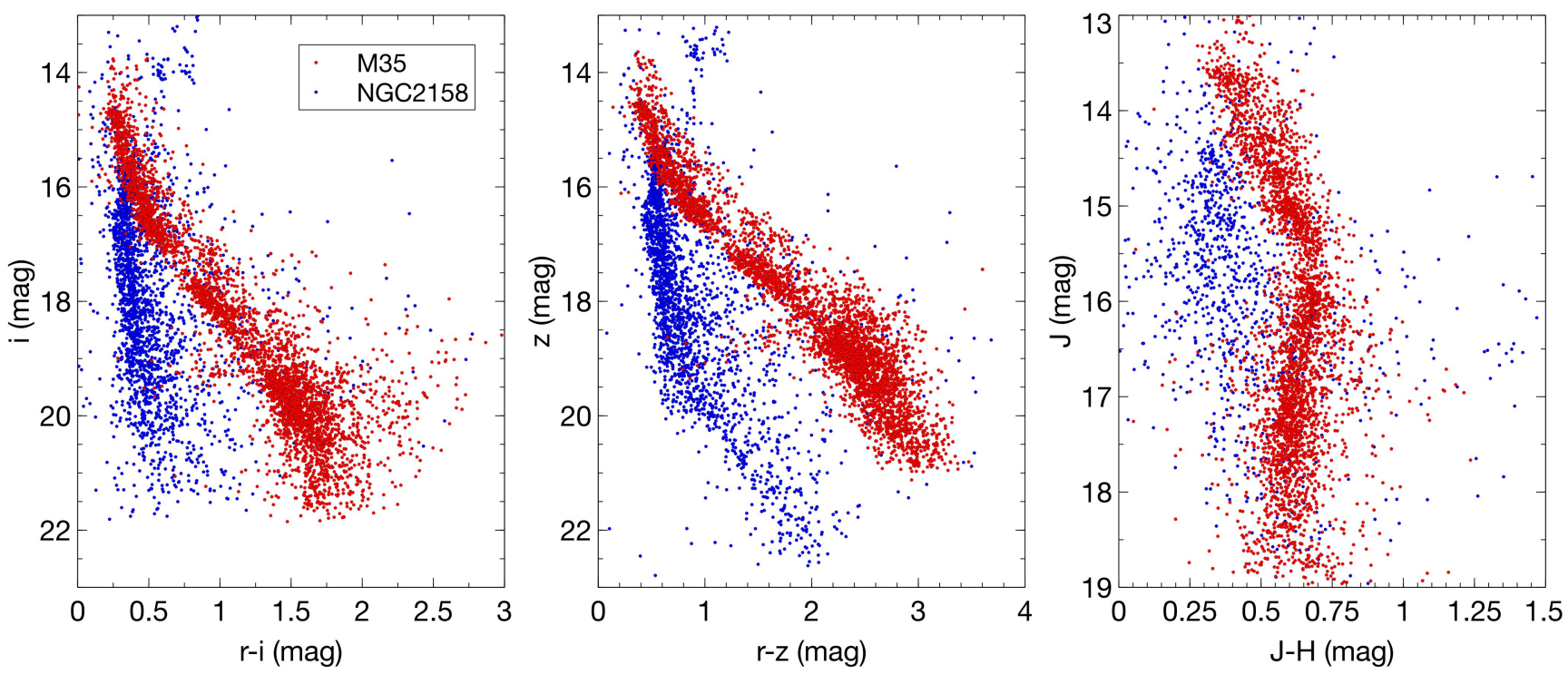

Fig. 5. Color-magnitude diagrams of the M 35 high probability members ( $\geq 0.5$. red dots) and of all the sources within $3^{\prime}$ of NGC 2158 center (blue dots).

baseline and typically reach a depth of $21 \approx 22$ mag in the optical and $\approx 19$ mag in the near-infrared, while saturation arises around $12 \sim 13 \mathrm{mag}$ at the bright end. This data set allows us to derive multiwavelength photometry from the optical to the nearinfrared for 338892 sources, and proper motions for 194452 of them. We use these measurements to derive the membership probability of all the sources with a proper motion measurement. We obtain:

- 4349 candidate members with probability greater than $50 \%$. - 1726 candidate members with probability greater than $75 \%$. - 305 candidate members with probability greater than $95 \%$.

These numbers are consistent with the census presented in Barrado y Navascués et al. (2001) over a smaller area. Contamination is expected to be significant as the locus of the cluster in the proper motion, color-magnitude and color-color diagrams overlaps largely with the field and background locus. This new study nevertheless provides a coherent and quantitative membership analysis of M 35 based on a large fraction of the best ground-based data sets obtained over the past 18 years.

Acknowledgements. H. Bouy is funded by the Ramón y Cajal fellowship program number RYC-2009-04497. This research has been funded by Spanish grants AYA2012-38897-C02-01. E. Moraux ackowledges funding from the Agence Nationale pour la Recherche program ANR 2010 JCJC 05011 "DESC (Dynamical Evolution of Stellar Clusters)". A. Ribas is funded by ESAC Science Operations Division research funds with code SC 1300016149. Based on observations obtained with MegaPrime/MegaCam, a joint project of CFHT and CEA/DAPNIA, at the Canada-France-Hawaii Telescope (CFHT) which is operated by the National Research Council (NRC) of Canada, the Institut National des Sciences de l'Univers of the Centre National de la Recherche Scientifique (CNRS) of France, and the University of Hawaii. Based on observations obtained with WIRCam, a joint project of CFHT, Taiwan, Korea, Canada, France, at the Canada-France-Hawaii Telescope (CFHT). This paper makes use of data obtained from the Isaac Newton Group Archive which is maintained as part of the CASU Astronomical Data Centre at the Institute of Astronomy, Cambridge. The data was made publically available through the Isaac Newton Group's Wide Field Camera Survey Programme. The Isaac Newton Telescope is operated on the island of La Palma by the Isaac Newton Group in the Spanish Observatorio del Roque de los Muchachos of the Instituto de Astrofísica de Canarias. This research used the facilities of the Canadian Astronomy Data Centre operated by the National Research Council of Canada with the support of the Canadian Space Agency. This research draws upon data distributed by the NOAO Science Archive. NOAO is operated by the Association of Universities for Research in Astronomy (AURA) under cooperative agreement with the National Science Foundation. This publication makes use of data products from the Two Micron All Sky Survey, which is a joint project of the University of Massachusetts and the Infrared Processing and Analysis Center/California Institute of Technology, funded by the National Aeronautics and Space Administration and the National Science Foundation. This work is based in part on data obtained as part of the UKIRT Infrared Deep Sky Survey. This research has made use of the VizieR and Aladin images and catalog access tools and of the SIMBAD database, operated at CDS, Strasbourg, France. This research made use of data from the SDSS survey. Funding for the creation and distribution of the SDSS Archive has been provided by the Alfred P. Sloan Foundation, the Participating Institutions, the National Aeronautics and Space Administration, the National Science Foundation, the US Department of Energy, the Japanese Monbukagakusho, and the Max Planck Society. The SDSS Web site is http://www.sdss.org/. The Participating Institutions are The University of Chicago, Fermilab, the Institute for Advanced Study, the Japan Participation Group, The Johns Hopkins University, the Max-Planck-Institute for Astronomy (MPIA), the Max-Planck-Institute for Astrophysics (MPA), New Mexico State University, Princeton University, the United States Naval Observatory, and the University of Washington. Based on data collected at Subaru Telescope and Kiso observatory (University of Tokyo) and obtained from the SMOKA, which is operated by the Astronomy Data Center, National Astronomical Observatory of Japan.

\section{References}

Ahn, C. P., Alexandroff, R., Allende Prieto, C., et al. 2012, ApJS, 203, 21

Autry, R. G., Probst, R. G., Starr, B. M., et al. 2003, in SPIE Conf. Ser. 4841, eds. M. Iye, \& A. F. M. Moorwood, 525

Barrado, D. 2001, in Highlights of Spanish astrophysics II, eds. J. Zamorano, J. Gorgas, \& J. Gallego, 161

Barrado, D., Deliyannis, C. P., \& Stauffer, J. R. 2001a, ApJ, 549, 452

Barrado, D., Stauffer, J. R., Bouvier, J., \& Martín, E. L. 2001b, ApJ, 546, 1006

Barrado, D., Stauffer, J. R., \& Bouvier, J. 2005, in The Initial Mass Function 50 Years Later, eds. E. Corbelli, F. Palla, \& H. Zinnecker, Astrophys. Space Sci. Lib., 327, 133

Barrado y Navascués, D., Stauffer, J. R., Bouvier, J., \& Martín, E. L. 2001, ApJ, 546, 1006

Bayo, A., Barrado, D., Stauffer, J., et al. 2011, A\&A, 536, A63

Bertin, E. 2006, in Astronomical Data Analysis Software and Systems XV, eds. C. Gabriel, C. Arviset, D. Ponz, \& S. Enrique, ASP Conf. Ser., 351, 112

Bertin, E. 2011, in Astronomical Data Analysis Software and Systems XX, eds. I. N. Evans, A. Accomazzi, D. J. Mink, \& A. H. Rots, ASP Conf. Ser., 442, 435

Bertin, E., \& Arnouts, S. 1996, A\&AS, 117, 393

Boulade, O., Charlot, X., Abbon, P., et al. 2003, in SPIE Conf. Ser. 4841, eds.

M. Iye, \& A. F. M. Moorwood, 72

Bouy, H., Bertin, E., Moraux, E., et al. 2013, A\&A, 554, A101 
A\&A 575, A120 (2015)

Carraro, G., Girardi, L., \& Marigo, P. 2002, MNRAS, 332, 705

Cuillandre, J.-C., Luppino, G. A., Starr, B. M., \& Isani, S. 2000, in SPIE Conf. Ser. 4008, eds. M. Iye, \& A. F. Moorwood, 1010

Drew, J. E., Greimel, R., Irwin, M. J., et al. 2005, MNRAS, 362, 753

Geller, A. M., Mathieu, R. D., Braden, E. K., et al. 2010, AJ, 139, 1383

Howell, S. B., Sobeck, C., Haas, M., et al. 2014, PASP, 126, 398

Itoh, N., Soyano, T., Tarusawa, K., et al. 2001, Publications of the National Astronomical Observatory of Japan, 6, 41

Ives, D. 1998, IEEE Spectrum, 16, 20

Kalirai, J. S., Fahlman, G. G., Richer, H. B., \& Ventura, P. 2003, AJ, 126, 1402

Leonard, P. J. T., \& Merritt, D. 1989, ApJ, 339, 195

McNamara, B. J., Harrison, T. E., McArthur, B. E., \& Benedict, G. F. 2011, AJ, 142,53

Meibom, S., Mathieu, R. D., \& Stassun, K. G. 2009, ApJ, 695, 679
Metzger, M. R., Luppino, G. A., \& Miyazaki, S. 1995, BAAS, 27, 1389 Miyazaki, S., Komiyama, Y., Sekiguchi, M., et al. 2002, PASJ, 54, 833 Puget, P., Stadler, E., Doyon, R., et al. 2004, in SPIE Conf. Ser. 5492, eds. A. F. M. Moorwood, \& M. Iye, 978

Reimers, D., \& Koester, D. 1988, A\&A, 202, 77

Sarro, L. M., Bouy, H., Berihuete, A., et al. 2014, A\&A, 563, A45

Skrutskie, M. F., Cutri, R. M., Stiening, R., et al. 2006, AJ, 131, 1163

Soderblom, D. R., Jones, B. F., Balachandran, S., et al. 1993, AJ, 106, 1059

Steinhauer, A., \& Deliyannis, C. P. 2004, ApJ, 614, L65

Sung, H., \& Bessell, M. S. 1999, MNRAS, 306, 361

Vandame, B. 2002, in Astronomical Data Analysis II, eds. J.-L. Starck, \& F. D. Murtagh, Proc. SPIE Conf., 4847, 123

Vidal, N. V. 1973, A\&AS, 11, 93

Wolfe, T., Armandroff, T., Blouke, M. M., et al. 2000, in SPIE Conf. Ser. 3965 eds. M. M. Blouke, N. Sampat, G. M. Williams, \& T. Yeh, 80 